\title{
THE CARATHÉODORY-CARTAN-KAUP-WU THEOREM ON AN INFINITE-DIMENSIONAL HILBERT SPACE
}

\author{
JOSEPH A. CIMA, IAN GRAHAM*, KANG TAE KIM ${ }^{\dagger}$ AND \\ STEVEN G. KRANTZ
}

\begin{abstract}
This paper treats a holomorphic self-mapping $f: \Omega \rightarrow \Omega$ of a bounded domain $\Omega$ in a separable Hilbert space $\mathcal{H}$ with a fixed point $p$. In case the domain is convex, we prove an infinite-dimensional version of the CartanCarathéodory-Kaup-Wu Theorem. This is basically a rigidity result in the vein of the uniqueness part of the classical Schwarz lemma. The main technique, inspired by an old idea of $\mathrm{H}$. Cartan, is iteration of the mapping $f$ and its derivative. A normality result for holomorphic mappings in the compact-weakopen topology, due to Kim and Krantz, is used.
\end{abstract}

\section{$\S 0$. Introduction}

Perhaps the most important part of the classical Schwarz lemma is the uniqueness statement: If $f: D \rightarrow D$ is a holomorphic function from the unit disc $D$ to itself, $f(0)=0$, and $\left|f^{\prime}(0)\right|=1$, then $f$ is a rotation. This rigidity statement has had considerable effect in the subject of complex differential geometry. It is the wellspring of many holomorphically invariant metrics, and has had a notable influence in the subject of mapping theory.

The analogous result in higher (finite) dimensions has been explored by Carathéodory, Cartan, Kaup, and Wu. See [KRA] and [WU] for a careful discussion of the matter. The theorem is that, if a holomorphic mapping $f: \Omega \rightarrow \Omega$ of a bounded domain $\Omega$ in $\mathbb{C}^{n}$ satisfies $f(p)=p$ for some $p \in \Omega$ and $|\operatorname{det}(D f(p))|=1$, then $f$ is a biholomorphic mapping. We remark here

\section{Received April 1, 2005.}

Revised September 2, 2005.

2000 Mathematics Subject Classification: Primay46G20, Secondary 32H50.

*Author supported in part by the Natural Sciences and Engineering Research Council of Canada Grant \# A9221.

${ }^{\dagger}$ Author supported in part by the grant KRF-2002-070-C00005 of The Korea Research Foundation.

${ }^{\ddagger}$ Author supported in part by NSF Grant DMS-9988854. 
that the condition on the Jacobian determinant is, in this case, equivalent to saying that every eigenvalue of the Jacobian matrix has modulus 1.

In recent years, the study of holomorphic functions and mappings on a Hilbert or Banach space has received much attention. Work of Lempert (see for instance [LEM]) has served as a catalyst to this activity. The more recent work of Kim/Krantz (see [KIK1], [KIK2]) is the more direct genesis of the present paper. In this rather general setting, many of the familiar tools of finite-dimensional analysis are no longer available. The geometry is much more difficult. Yet there is interest in developing these ideas because of potential applications to mathematical physics and partial differential equations.

This paper develops a version of the Carathéodory-Cartan-Kaup-Wu theorem on a separable Hilbert space $\mathcal{H}$. We begin by formulating and proving a result on a convex domain $\Omega \subseteq \mathcal{H}$. This is Theorem 1.1. Afterward, in Section 3, we present a more refined result (Proposition 3.1) on the ball in a separable Hilbert space.

The authors thank the Banff International Research Station (BIRS) for hosting us during the work on this problem, and for providing a stimulating working environment. We thank John McCarthy, Peter Rosenthal and Warren Wogen for advice and information about analysis on Hilbert spaces.

\section{$\S 1$. Statement of the main results}

Let $\mathcal{H}$ be a separable, complex Hilbert space, and let $B$ denote its open unit ball. Let $S^{1}$ represent the unit circle in the complex plane $\mathbb{C}$. For a bounded linear operator $T$ on $\mathcal{H}$, denote by $\sigma(T)$ its spectrum, i.e.

$$
\sigma(T)=\{\lambda \in \mathbb{C} \mid T-\lambda I \text { is not invertible }\} .
$$

A bounded linear operator $T: \mathcal{H} \rightarrow \mathcal{H}$ is said to be triangularizable if there exists a basis $\left\{e_{1}, e_{2}, \ldots\right\}$ such that $T\left(\mathbb{C} e_{1}+\ldots+\mathbb{C} e_{N}\right) \subset \mathbb{C} e_{1}+$ $\ldots+\mathbb{C} e_{N}$ for every positive integer $N$. In this case, we shall sometimes say that $T$ is upper-triangular with respect to the basis $\left\{e_{1}, e_{2}, \ldots\right\}$. Note that the basis that renders an operator upper-triangular can always be taken to be orthonormal, just because the Gram-Schmidt process preserves the flags $E_{N}=\mathbb{C} e_{1}+\cdots+\mathbb{C} e_{N}$.

Now we formulate the principal result of this paper:

TheOREm 1.1. Let $\Omega \subseteq \mathcal{H}$ be a bounded convex domain. Fix a point $p \in \Omega$. Let $f: \Omega \rightarrow \Omega$ be a holomorphic mapping such that 
(a) $f(p)=p$;

(b) the differential $d f_{p}$ is triangularizable ;

(c) $\sigma\left(d f_{p}\right) \subseteq S^{1}$.

Then $f$ is a biholomorphic mapping.

To put the theorem in perspective, notice that in dimension $n=1$ the hypothesis specializes to just $\left|f^{\prime}(p)\right|=1$. In several (but finitely many) variables, the hypothesis is equivalent to (i) non-degeneracy of the Jacobian matrix at $p$ and (ii) all eigenvalues of the Jacobian having modulus 1. In the finite-dimensional case, one can always triangularize. In infinite dimensions there are geometric conditions (involving the Fredholm index) for triangularization.

Even if $T$ is a unitary operator on a Hilbert space $\mathcal{H}$ (so that certainly $\sigma(T) \subseteq S^{1}$ ), it does not necessarily follow that $T$ is triangularizable. For example, take $T$ to be the forward shift on $\ell^{2}(\mathbb{Z})$. See [HER] for more on the triangular operators.

Concerning the assumption (b) in Theorem 1.1, it seems appropriate to present an example of a bounded convex domain with an automorphism whose derivative at a fixed point is upper-triangular. Consider the map $A: \mathbb{C}^{2} \rightarrow \mathbb{C}^{2}$ defined by $A(z, w)=(z+b w,-w)$ for an arbitrary choice for $b \in \mathbb{C} \backslash\{0\}$. Then $A$ is an involution (i.e. $A \circ A=I$ ) with eigenvalues \pm 1 ; also $A$ is an upper-triangular linear map. Take any bounded domain $D$ in $\mathbb{C}^{2}$ containing the origin. Let

$$
V=D \cup A(D) .
$$

Then let $\Omega$ be the convex hull of $V$ in $\mathbb{C}^{2}$. It is obviously a bounded convex domain containing the origin. Then $A$ is an automorphism of $\Omega$ satisfying $A(0)=0$. Since $d A_{0}=A$, the differential $d A_{0}$ is upper-triangular. Furthermore, if one would sacrifice the convexity of the domain, it is possible also to obtain a non-linear example. Let $G(z, w)=\left(z, w+z^{2}\right)$ for instance, let $f=G \circ A \circ G^{-1}$, and let $W=G(\Omega)$. Then $W$ is a bounded domain in $\mathbb{C}^{2}$, and $f$ is an automorphism of $W$ with $f(0)=0$. We see that $d f_{0}$ is clearly upper-triangularizable. It is simple to modify this example to give an example in an infinite dimensional Hilbert space; one simply needs to consider a map that is equal to $A$ on a two-dimensional subspace and the identity on the orthogonal complement of this subspace. 
The referee has raised the question of how large is the class of holomorphic mappings $f: \Omega \rightarrow \Omega$ satisfying the conditions (a), (b) and (c) of Theorem 1.1, within the class of such mappings satisfying only (a) and (c). We believe that rigidity phenomena for automorphisms of domains in infinite dimensions would imply that the answer depends very much on the domain. A related question is how large is the class of triangularizable operators on a separable Hilbert space $H$ within the class of all bounded linear mappings of $H$. In this connection we mention the Weyl-von Neumann-Berg theorem [DAV, p. 59], which asserts that every normal operator is a small compact perturbation of a diagonalizable (in particular triangularizable) operator. We thank the referee and the editors for their remarks.

\section{§2. Proof of Theorem 1.1}

The proof has several steps, some of which are of independent interest.

\subsection{Basic facts on the differential}

Let $\Omega_{1}$ and $\Omega_{2}$ be domains in the separable Hilbert space $\mathcal{H}$ and let $f: \Omega_{1} \rightarrow \Omega_{2}$ be a holomorphic mapping. The differential of $f$ at a point $p \in \Omega_{1}$ is the bounded linear operator on $\mathcal{H}$ defined by

$$
d f_{p}(v)=\lim _{\mathbb{C} \ni h \rightarrow 0} \frac{f(p+h v)-f(p)}{h} .
$$

Write $B(p ; r)=\{z \in \mathcal{H} \mid\|z-p\|<r\}$. Suppose that $\Omega_{2}$ is bounded, say $\Omega_{2} \subseteq B(0 ; M)$. In this situation we have

Lemma 2.1. If $p \in \Omega_{1}$ and $\operatorname{dist}\left(p, \partial \Omega_{1}\right)=d>0$, then

$$
\left\|d f_{p}\right\| \leq \frac{M}{d}
$$

Proof. Choose $\rho>0$ so that $\bar{B}(p ; \rho) \subset \Omega_{1}$. Let $v$ be a unit vector in $\mathcal{H}$. The integral representation

$$
d f_{p}(v)=\frac{1}{2 \pi i} \int_{|\zeta|=\rho} \frac{f(p+\zeta v)}{\zeta^{2}} d \zeta
$$

leads immediately to the Cauchy estimates

$$
\left\|d f_{p}(v)\right\| \leq \frac{M}{\rho}
$$

and we may let $\rho$ tend to $d$. 
Lemma 2.2. If $\left\|d f_{z}\right\| \leq A$ for every $z \in B(p ; r)$, then for any pair $z, w \in B(p ; r)$ one has

$$
\|f(z)-f(w)\|<2 r A
$$

Proof. Write

$$
f(z)-f(w)=\int_{0}^{1} \frac{d}{d t}[f((1-t) w+t z)] d t
$$

and use the chain rule.

Now, in the situation of Theorem 1.1, $f$ is a holomorphic self-map of a bounded convex domain in $\mathcal{H}$ and $p$ is a fixed point. Let us write $T=d f_{p}$.

We are assuming that $T$ is triangularizable. Hence it is possible to choose a basis $e_{1}, e_{2}, \ldots$ such that

$$
T\left(\mathbb{C} e_{1}+\cdots+\mathbb{C} e_{N}\right) \subset \mathbb{C} e_{1}+\cdots+\mathbb{C} e_{N}
$$

for every positive integer $N$. For convenience set

$$
E_{N}=\mathbb{C} e_{1}+\cdots+\mathbb{C} e_{N}
$$

for every positive integer $N$. We shall call such $E_{N}$ a flag. The union of these flags yields a vector space that is dense in $\mathcal{H}$.

From here on we assume that the operator $T$ is upper-triangular with respect to a fixed orthonormal basis $e_{1}, e_{2}, \ldots$

Notice that $\sigma(T)$ is contained in the unit circle. Since $T$ is uppertriangular, the diagonal entries are contained in its spectrum $\sigma(T)$, and hence are of modulus 1.

We also note the following consequence of Lemma 2.1:

COROLlary 2.3. There exists a constant $C$ such that

$$
\left\|T^{m}\right\| \leq C
$$

for every positive integer $m$. 


\subsection{Iteration of $T$}

In this section $T$ is an upper triangular matrix whose spectrum $\sigma(T)$ is contained in $S^{1}$ and whose positive powers are uniformly bounded in norm. Denote by $\lambda_{j}$ the $(j, j)$-th diagonal entry of $T$. Then $\left|\lambda_{j}\right|=1, j \in \mathbb{N}$.

Lemma 2.4. There exists a sequence of natural numbers $\{m(k)\}_{k}$ such that for each fixed $j \in \mathbb{N}, \lambda_{j}^{m(k)} \rightarrow 1$ as $k \rightarrow \infty$.

Proof. The sequence of powers $\left\{\lambda_{1}^{k}\right\}$ is bounded, so there is a convergent subsequence, say $\lambda_{1}^{m(1, k)} \rightarrow \alpha_{1}$ as $k \rightarrow \infty$, where $\left|\alpha_{1}\right|=1$. Similarly

the sequence of powers $\left\{\lambda_{2}^{m(1, k)}\right\}$ is bounded, so there is a subsequence $\{m(2, k)\}$ of $\{m(1, k)\}$ such that $\lambda_{2}^{m(2, k)} \rightarrow \alpha_{2}$ as $k \rightarrow \infty$, where $\left|\alpha_{2}\right|=1$. Continuing in this way and using a diagonal sequence argument, we obtain a subsequence $\{m(k, k)\}$ of the natural numbers and complex numbers $\alpha_{j}$, $j \in \mathbb{N}$, of modulus one such that $\lambda_{j}^{m(k, k)} \rightarrow \alpha_{j}$ as $k \rightarrow \infty$ for each $j \in \mathbb{N}$. Now the sequence

$$
m(k)=m(k+1, k+1)-m(k, k), \quad k \in \mathbb{N}
$$

is easily seen to have the property that

$$
\lambda_{j}^{m(k)} \rightarrow \frac{\alpha_{j}}{\alpha_{j}}=1
$$

as $k \rightarrow \infty$, for each $j \in \mathbb{N}$.

If $A$ is an infinite square matrix we denote the $(i, j)$-th entry by $A_{i, j}$, $1 \leq i, j \leq \infty$. Also, we order the positions above the diagonal in such a matrix first by column and then by row, i.e. the ordering is $(1,2),(1,3)$, $(2,3),(1,4) \ldots$. If $N$ is a positive integer, we denote the $N \times N$ truncation of such a matrix by $A_{N}$. The $N \times N$ identity matrix will be denoted by $I_{N}$.

LEMMA 2.5. There is a sequence of natural numbers $\{\mu(k)\}_{k}$ such that, for each $N \in \mathbb{N}$,

$$
\left(T_{N}\right)^{\mu(k)} \rightarrow I_{N}, \quad k \rightarrow \infty
$$

Proof. Write $T=S+V$, where $S$ is a diagonal matrix with diagonal entries of modulus 1 and $V$ has zeros on and below the main diagonal. Denote the diagonal entries of $S$ by $\lambda_{j}, j=1,2, \ldots$. 
By Lemma 2.4, there exists a sequence of natural numbers $\{m(k)\}$ such that $\lambda_{j}^{m(k)} \rightarrow 1$ as $k \rightarrow \infty$, for each fixed $j$.

The entries $\left\{\left(T^{m(k)}\right)_{1,2}\right\}_{k}$ are bounded, so there is a subsequence $\{\mu(k ; 1,2)\}$ of $\{m(k)\}$ such that $\left\{\left(T^{\mu(k ; 1,2)}\right)_{1,2}\right\}_{k}$ converges. By similar reasoning, a further subsequence $\{\mu(k ; 1,3)\}$ has the property that $\left\{\left(T^{\mu(k ; 1,3)}\right)_{1,3}\right\}_{k}$ converges, and a further subsequence of that one, denoted by $\left\{\mu(k ; 2,3\}\right.$, has the property that $\left\{\left(T^{\mu(k ; 2,3)}\right)_{2,3}\right\}_{k}$ converges. Continuing in this way and extracting a diagonal subsequence yields a subsequence $\{\mu(k)\}$ of $\{m(k)\}$ such that $\left.\left\{\left(T^{\mu(k)}\right)\right)_{i, j}\right\}_{k}$ converges for all $(i, j), \quad 1 \leq i<$ $j<\infty$.

Thus there is an infinite square matrix $W$, whose entries on and below the main diagonal are zero, such that for each $N \in \mathbb{N}$ we have $\left(T_{N}\right)^{\mu(k)} \rightarrow$ $I_{N}+W_{N}$. (For fixed $N$ the convergence may be taken to be in norm, but the norm convergence is not uniform in $N$.)

Now choose the smallest value of $N \geq 2$ with the property that $W_{N} \neq 0$. Then at least one of the entries in the last column of $W_{N}$ is nonzero, and all entries in the other columns of $W_{N}$ are zero. If $\ell$ is a positive integer, then

$$
\left(\left(T_{N}\right)^{\mu(k)}\right)^{\ell}=\left(T_{N}\right)^{\mu(k) \ell} \rightarrow\left(I_{N}+W_{N}\right)^{\ell}, \quad k \rightarrow \infty,
$$

and the entries in the last column of the matrix on the right are given by $\ell$ times the corresponding entries in $\left(I_{N}+W_{N}\right)$ (except for a 1 in the $(N, N)$-position).

But this is a contradiction to the power boundedness of $T$ for sufficiently large $\ell$ (see Corollary 2.3). We conclude that no such $N$ exists, i.e. $W=0$. Therefore the sequence $\{\mu(k)\}$ has the property that $\left(T_{N}\right)^{\mu(k)} \rightarrow I_{N}$ as $k \rightarrow \infty$ for each fixed $N$.

\subsection{Iteration of $f$}

Next, consider the iteration given by $f^{1}=f, f^{m}=f \circ f^{m-1}$ for each integer $m>1$.

We need the following two fundamental results. There is in fact a Banach space version of the theorem of Kim and Krantz [KIK2]; we indicate a proof here for the Hilbert space case.

Theorem 2.6. (Kim/Krantz [KIK2]) Let $\Omega_{1}, \Omega_{2}$ be domains in a separable Hilbert space $\mathcal{H}$, and let $\Omega_{2}$ be bounded. Then every sequence $\left\{h_{n}\right.$ : $\left.\Omega_{1} \rightarrow \Omega_{2} \mid n=1,2, \ldots\right\}$ of holomorphic mappings admits a subsequence $\left\{h_{n(k)}\right\}_{k}$ that converges to a holomorphic mapping $\widehat{h}$ from $\Omega_{1}$ into the closed 
convex hull of $\Omega_{2}$, in the compact-weak-open topology (i.e., the compact-open topology in which the strong topology is used on the domain space and the weak topology is used on the target space).

Proof. Let $\langle\cdot, \cdot\rangle$ denote the Hilbert space inner product (linear in the first variable, conjugate-linear in the second). One needs to show that for every sequence $\left\{h_{n}: \Omega_{1} \rightarrow \Omega_{2} \mid n=1,2, \ldots\right\}$ of holomorphic mappings, there is a subsequence $\left\{h_{n(k)}\right\}_{k}$ and a holomorphic mapping $\widehat{h}: \Omega_{1} \rightarrow \mathcal{H}$ such that, for all $g$ in the unit ball of $\mathcal{H},\left\langle h_{n(k)}, g\right\rangle \rightarrow\langle\widehat{h}, g\rangle$, uniformly on compact subsets, as $k \rightarrow \infty$. If $g$ is such that $\Re\langle w, g\rangle<1$ for all $w \in \Omega_{2}$, then it is clear that $\Re\langle\widehat{h}, g\rangle \leq 1$, i.e. the image of $\widehat{h}$ must be contained in the closed convex hull of $\Omega_{2}$.

Let $\left\{z_{n}\right\}_{n \in \mathbb{N}}$ be a dense sequence in $\Omega_{1}$. We are going to do the usual diagonal sequence construction. The sequence $\left\{h_{n}\right\}$ has a subsequence $\left\{h_{n(1, k)}\right\}_{k}$ such that $h_{n(1, k)}\left(z_{1}\right)$ converges weakly to an element $\widehat{h}\left(z_{1}\right)$ as $k \rightarrow \infty$. This just uses the boundedness of $\Omega_{2}$.

Now choose a subsequence of $\{n(1, k)\}_{k}$, denoted by $\{n(2, k)\}_{k}$, so that $h_{n(2, k)}\left(z_{2}\right)$ converges weakly to an element $\widehat{h}\left(z_{2}\right)$ as $k \rightarrow \infty$. Continue and then choose the diagonal sequence $\left\{h_{n(k)}\right\}_{k}=\left\{h_{n(k, k)}\right\}_{k}$ generated by this process.

Since $\Omega_{2}$ is bounded, as we did earlier in the proof of Lemma 2.1, we let $M$ be a positive constant such that $\Omega_{2} \subset B(0 ; M)$.

Assume that $K$ is a compact subset of $\Omega_{1}$ and let $\epsilon>0$. Denote by $D=\operatorname{dist}\left(K, \partial \Omega_{1}\right)$ and let $\delta=\min \left\{\frac{D}{3}, \frac{D \epsilon}{18 M}\right\}$. Cover $K$ with a finite number of balls $B\left(x_{1} ; \delta\right), \ldots, B\left(x_{m} ; \delta\right)$ such that $B\left(x_{\ell} ; \delta\right) \cap K \neq \emptyset$ for any $\ell=1,2, \ldots, m$, where each $x_{\ell}$ belongs to the dense sequence $\left\{z_{n}\right\}_{n \in \mathbb{N}}$. Note that for $z \in B\left(x_{\ell} ; \delta\right)$ one has $\operatorname{dist}\left(z, \partial \Omega_{1}\right)>D / 3$. By Lemma 2.1 we have $\left\|\left.d h_{n}\right|_{z}\right\|<\frac{3 M}{D}$ for such $z$. This holds for every $n$. By Lemma 2.2 and the choice of $\delta$, we have

$$
\left\|h_{n}(z)-h_{n}(w)\right\|<\frac{6 M \delta}{D} \leq \frac{\epsilon}{3}
$$

for all $z, w \in B\left(x_{\ell}, \delta\right)$. For any $g$ in the unit ball of $H$, choose $J=J(g)$ so that, for $j, k>J$, we have

$$
\left|\left\langle h_{n(j)}\left(x_{\ell}\right)-h_{n(k)}\left(x_{\ell}\right), g\right\rangle\right|<\frac{\epsilon}{3}
$$

for $1 \leq \ell \leq m$. 
Now for any choice of $z \in K$ we have the existence of some $\ell$ with $z \in B\left(x_{\ell}, \delta\right)$. Then for $j, k>J$ we have

$$
\begin{aligned}
\left|\left\langle h_{n(j)}(z)-h_{n(k)}(z), g\right\rangle\right| \leq \mid & \left.h_{n(j)}(z)-h_{n(j)}\left(x_{\ell}\right), g\right\rangle \mid \\
& +\left|\left\langle h_{n(j)}\left(x_{\ell}\right)-h_{n(k)}\left(x_{\ell}\right), g\right\rangle\right| \\
& +\left|\left\langle h_{n(k)}\left(x_{\ell}\right)-h_{n(k)}(z), g\right\rangle\right| .
\end{aligned}
$$

Each of the terms on the right hand side is less than $\epsilon / 3$. For the first and third terms this follows from (2), and for the second term it follows from the choice of $J$.

This shows that the sequence $h_{n(k)}(z)$ converges weakly to the "assignment" $\widehat{h}(z)$ uniformly on compacta. It remains only to show that the assignment is an analytic mapping on $\Omega_{1}$. But given a fixed $z_{0} \in \Omega_{1}$ and a unit vector $v \in \mathcal{H}$, we can find $a>0$ such that the closed disc

$$
S=\left\{z_{0}+\zeta v|| \zeta \mid \leq a\right\}
$$

is contained in $\Omega_{1}$, and hence the analytic functions $\left\langle h_{n(k)}\left(z_{0}+\zeta v\right), g\right\rangle$ converge uniformly to $\left\langle\widehat{h}\left(z_{0}+\zeta v\right), g\right\rangle$. The mapping $\zeta \mapsto \widehat{h}\left(z_{0}+\zeta v\right)$ from the disc of radius $a$ in $\mathbb{C}$ into $\mathcal{H}$ is therefore holomorphic. This says precisely that $\widehat{h}$ is Gateaux holomorphic [HIP], [MUJ]. Since a bounded Gateaux holomorphic mapping is holomorphic, we are done.

Remark 2.1. By the Cauchy estimates, it follows from Theorem 2.6 that the derivative $\left.d h_{n(k)}\right|_{z}(v)$ converges to $d \widehat{h}_{z}(v)$ weakly, uniformly on compact subsets of $\Omega_{1} \times \mathcal{H}$ (i.e., $z \in \Omega_{1}$ and $v \in \mathcal{H}$ ).

To see this, let $L$ be a compact subset of $\Omega_{1} \times \mathcal{H}$. Then for all $(z, v) \in L$, we have $\operatorname{dist}\left(z, \partial \Omega_{1}\right) \geq a>0$ and $\|v\| \leq b$, for some positive constants $a$ and $b$. It is elementary to see that there exists $r>0$ such that

$$
K=\{z+\zeta v|(z, v) \in L,| \zeta \mid \leq r\}
$$

is a compact subset of $\Omega_{1}$. Now the relation (1) gives

$$
\left.d h_{n(k)}\right|_{z}(v)-d h_{z}(v)=\frac{1}{2 \pi i} \int_{|\zeta|=r} \frac{h_{n(k)}(z+\zeta v)-h(z+\zeta v)}{\zeta^{2}} d \zeta,
$$

for all $(z, v) \in L$. Hence for any linear functional $\tau$ on $\mathcal{H}$,

$$
\left.\tau \circ d h_{n(k)}\right|_{z}(v)-\tau \circ d h_{z}(v)
$$




$$
=\frac{1}{2 \pi i} \int_{|\zeta|=r} \frac{\tau \circ h_{n(k)}(z+\zeta v)-\tau \circ h(z+\zeta v)}{\zeta^{2}} d \zeta .
$$

The assertion follows easily from this in view of the compactness of $K$.

Theorem 2.7. (H. Cartan) Let $\Omega$ be a bounded domain in $\mathcal{H}$ and let $p \in \Omega$. Let $f: \Omega \rightarrow \Omega$ be a holomorphic mapping with $f(p)=p$ and $d f_{p}=I$. Then $f$ coincides with the identity mapping of $\Omega$.

See [FRV], [KRA] for the proof of Cartan's theorem.

Now apply Theorem 2.6 to the sequence $\left\{f^{\mu(k)}\right\}$, where $f$ is the mapping in Theorem 1.1 and $\{\mu(k)\}$ is the sequence constructed in Lemma 2.5. We obtain a subsequence $\left\{f^{\nu(k)}\right\}$ that converges to some $\widehat{f}$ in the compact-weakopen-topology. The sequence of derivatives at $p$ is $\left\{T^{\nu(k)}\right\}$. The discussion of the preceding section shows that

$$
\lim _{k \rightarrow \infty}\left(T_{N}\right)^{\nu(k)}=I_{N}
$$

for every positive integer $N$.

Thus

$$
\left.d \widehat{f}_{p}\right|_{E_{N}}=I_{E_{N}} \text { for every } N=1,2, \ldots .
$$

Note that $\bigcup_{N} E_{N}$ is dense in $\mathcal{H}$. Since $d \widehat{f}_{p}$ is bounded and $\left.d \widehat{f}_{p}\right|_{E_{N}}=I_{N}$ for all $N$, it follows that $d \widehat{f_{p}}=I$.

Finally, the fact that $\widehat{f}(p)=p$ together with the convexity of $\Omega$ implies that $\widehat{f}(\Omega) \subseteq \Omega$. Now Cartan's theorem (Theorem 2.7) implies that $\widehat{f} \equiv$ id.

\subsection{Proof of Theorem 1.1}

It is time to complete the proof of Theorem 1.1. From the last part of the preceding subsection, we have

$$
\lim _{k \rightarrow \infty} f\left(f^{\nu(k)-1}(z)\right)=z=\lim _{k \rightarrow \infty} f^{\nu(k)-1}(f(z))
$$

in the compact-weak-open topology on $\Omega$.

By Theorem 2.6, choose a subsequence $\left\{f_{\ell}\right\}$ of $\left\{f^{\nu(k)-1}\right\}$ that converges to a holomorphic mapping $\widehat{h}: \Omega \rightarrow \Omega$ in the compact-weak-open topology. (Recall that $\Omega$ is convex and $\widehat{h}(p)=p$ ).

The second identity in (3) implies that

$$
\widehat{h} \circ f=\mathrm{id} \text {. }
$$


We see therefore that the holomorphic mapping $\widehat{h}$ is a left inverse of $f$, and that $f \circ \widehat{h}$ is a holomorphic mapping from $\Omega$ into itself.

On the other hand, one cannot immediately deduce from the first identity in (3) that $f \circ \widehat{h}=\mathrm{id}$, because it is only known at this point that $f_{\ell}$ converges to $\widehat{h}$ weakly. (With respect to the weak topology on the sourcedomain and the strong topology on the target-domain, holomorphic mappings need not be continuous.) So it is necessary to show that $f \circ \widehat{h}=\mathrm{id}$.

Now (4) implies that

$$
\widehat{d h}_{p} \circ d f_{p}=I .
$$

Since $d f_{p}$ is invertible, we see that $d \widehat{h}_{p}$ is also the right inverse of $d f_{p}$. This implies that

$$
d(f \circ \widehat{h})_{p}=d f_{p} \circ \widehat{d h}_{p}=I .
$$

Applying Cartan's Theorem (Theorem 2.7) again to $f \circ \widehat{h}: \Omega \rightarrow \Omega$, we see that

$$
f \circ \widehat{h}=\mathrm{id} .
$$

Therefore $f$ is a biholomorphic mapping of $\Omega$ onto itself. This completes the proof.

\section{§3. Closing remarks}

For holomorphic functions (i.e. $\mathbb{C}$-valued functions) on a domain in a separable Banach space, there is a normality result for the compact-open topology, similar to the finite-dimensional case [HUY], [KIK2], [MUJ]. For holomorphic mappings there is no such result unless one makes further restrictions [HUY]. However, it is possible to obtain interesting theorems about holomorphic mappings using normality with respect to the compactweak-open topology.

The assumption that the differential be triangularizable at the fixed point is not necessary for the conclusion of Theorem 1.1 to be valid. In the case of the unit ball $B$ of the Hilbert space $\mathcal{H}$, a unitary map conjugated by a Möbius transformation is a holomorphic automorphism, but in general the differential at the fixed point is not triangularizable. This phenomenon reflects the difficulty in the case of infinite dimensional holomorphy caused by the excessive size of the isotropy group in such cases as the ball (in finite dimensions, large isotropy group characterizes the ball - see [GRK]). However, the ball case has a reasonable formulation as follows. 
Proposition 3.1. Let $f: B \rightarrow B$ be a holomorphic mapping, let $p \in$ $B$, and let $M_{p}$ be a Möbius transformation of the ball sending $p$ to the origin. If $f$ satisfies:

(1) $f(p)=p$,

(2) $\left.\left.U \circ d\left[M_{p}\right]\right|_{p} \circ d f_{p} \circ d\left[M_{p}^{-1}\right]\right|_{0}$ is triangularizable, for some unitary transform $U$, and

(3) $\sigma\left(\left.\left.U \circ d\left[M_{p}\right]\right|_{p} \circ d f_{p} \circ d\left[M_{p}^{-1}\right]\right|_{0}\right)$ lies in the unit circle in $\mathbb{C}$,

then $f$ is an automorphism of $B$.

The arguments given in this article surely yield a proof of Proposition 3.1. However, one can simplify the argument, thanks to the fact that the domain in consideration is the unit ball. Indeed, from Schwarz's lemma, upper triangularity and the spectrum condition, one obtains that the operator $V=\left.\left.U \circ d\left[M_{p}\right]\right|_{p} \circ d f_{p} \circ d\left[M_{p}^{-1}\right]\right|_{0}$ has norm 1. It is known [DIN] that such a linear transformation $V$ is in fact unitary. Thus Cartan's Theorem applied to $V^{-1} \circ U \circ M_{p} \circ f \circ M_{p}^{-1}$ implies that this map is the identity map. Hence $f$ is a holomorphic automorphism of $B$. The authors would like to thank Warren Wogen for pointing out this line of reasoning.

The convexity assumption on $\Omega$ was necessary due to the use of weak convergence in several places. Whether one can remove this additional assumption should be an interesting problem to explore in future work. It would also be of interest to know whether there is a result of Schwarz-Pick type in our infinite-dimensional context.

\section{REFERENCES}

[BOM] S. Bochner and W. T. Martin, Functions of Several Complex Variables, Princeton University Press, Princeton, 1936.

[CON] J. B. Conway, A Course in Operator Theory, American Mathematical Society, Providence, RI, 2000.

[DAV] K. R. Davidson, $C^{*}$-algebras by example, Fields Institute Monographs, American Mathematical Society, Providence, RI, 1996.

[DIN] S. Dineen, The Schwarz Lemma, The Clarendon Press, Oxford University Press, Oxford, 1989

[DUS] N. Dunford and J. T. Schwartz, Linear Operators, Interscience, New York, 1988.

[FRV] T. Franzoni and E. Vesentini, Holomorphic Maps and Invariant Distances, NorthHolland, Amsterdam, 1980. 
[GRK] R. E. Greene and S. G. Krantz, Characterization of complex manifolds by the isotropy subgroups of their automorphism groups, Indiana Univ. Math. J., 34 (1985), 865-879.

[HER] D.A. Herrero, Triangular operators, Bull. London Math. Soc., 23 (1991), 513-554.

[HIP] E. Hille and R. S. Phillips, Functional Analysis and Semigroups, Amer. Math. Soc. Coll. Publ. 31, Providence, R. I., 1957.

[HUY] C.-G. Hu and T.-H. Yue,Normal families of holomorphic mappings, J. Math. Anal. Appl., 171(1992), 436-447.

[KIK1] K.-T. Kim and S. G. Krantz, Characerization of the Hilbert ball by its automorphism group, Trans. Amer. Math. Soc., 354 (2002), 2797-2818.

[KIK2] K.-.T. Kim and S. G. Krantz, Normal families of holomorphic functions and mappings on a Banach space, Expo. Math., 21 (2003), 193-218.

[KRA] S. G. Krantz, Function Theory of Several Complex Variables, American Mathematical Society-Chelsea, Providence, RI, 2001.

[LEM] L. Lempert, The Dolbeault complex in infinite dimensions, J. Amer. Math. Soc., 11 (1998), 485-520.

[MUJ] J. Mujica, Complex Analysis in Banach Spaces, North-Holland, Amsterdam and New York, 1986.

[NAR] R. Narasimhan, Several Complex Variables, University of Chicago Press, Chicago, 1971.

[WU] H. H. Wu, Normal families of holomorphic mappings, Acta Math., 119 (1967), $193-233$.

Joseph A. Cima

Department of Mathematics

University of North Carolina

Chapel Hill, North Carolina 27514 USA

cima@math.unc.edu

Ian Graham

Department of Mathematics

University of Toronto

Toronto, CANADA M5S $3 G 3$

graham@math.toronto.edu

Kang Tae Kim

Department of Mathematics

Pohang University of Science and Technology

Pohang 790-784 KOREA

kimkt@postech.ac.kr 
Steven G. Krantz

Department of Mathematics

Campus Box 1146

Washington University in St. Louis

St. Louis, Missouri 63130 USA

sk@math . wustl . edu 